(anti-angiogenic agent plus PARPi) in epithelial ovarian cancer (EOC) patients was approved in many previous studies, but there were few data in patients after PARPi progression. The aim of the Korean Gynecologic Oncology Group (KGOG) 3056/ NIRVANA-R trial is to investigate the efficacy of niraparib in combination with bevacizumab as a maintenance therapy in platinum-sensitive ovarian cancer patients who were previously treated with a PARPi.

Methods: The KGOG3056/NIRVANA-R is a multi-centre, investigator-initiated, single-arm, phase II trial recruiting recurrent EOC patients from seven KGOG sites. This study included patients with platinum-sensitive recurrent EOC who received at least two previous courses of platinum-containing therapy and had been treated with a PARPi. Mucinous histology type was excluded. Patients who had responded to the last platinum regimen (either complete or partial response) were eligible to participate in this study. Forty-four patients will be recruited and treated with niraparib and bevacizumab for maintenance therapy until disease progression, unacceptable toxicity, or withdrawal of patient consent. The primary endpoint is progression-free survival (PFS, 6-month PFS rate) according to the Response Evaluation Criteria in Solid Tumors guidelines. Accrual is expected to be completed in 2022, followed by presentation of results in 2023.

Poster (041)

Epithelial Ovarian Cancer including Borderline Tumor https://doi.org/10.3802/jgo.2021.32.S1.041

\section{Trial in progress: a phase II trial of lenvatinib plus pembrolizumab in recurrent gynecological clear cell adenocarcinomas (LARA) - APGOT OV3}

\author{
Silvana T Wijaya, ${ }^{1}$ Natalie YL Ngoi, ${ }^{1}$ Jessica Lee, ${ }^{1}$ Byoung-Gie Kim, ${ }^{2}$ \\ Jung-Yun Lee, ${ }^{3}$ David SP Tan ${ }^{1, *}$ \\ 'Department of Haematology-Oncology, National University Cancer \\ Institute Singapore, Singapore (David_sp_tan@nuhs.edu.sg) \\ ${ }^{2}$ Department of Obstetrics and Gynecology, Samsung Medical Center, \\ Sungkyunkwan University School of Medicine, Seoul, Korea \\ ${ }^{3}$ Department of Obstetrics and Gynecology, Yonsei University College of \\ Medicine, Seoul, Korea
}

Background: Advanced and recurrent clear cell gynecologic cancers (CCGCs) remain an area of significant unmet need. CCGCs are associated with lower response rates to platinumbased chemotherapy compared to high-grade serous ovarian cancers, and worse 5-year stage-adjusted disease-specific survival. CCGCs are more prevalent in East Asia, constituting up to $25 \%$ of all epithelial ovarian cancers. CCGCs bear remarkably similar gene expression profiles to clear cell renal cell cancers - they have an immune-suppressive microenvironment, prominent upregulation of HIF- $1 \alpha$ and a hypoxia-like mRNA expression signature. This provides rationale for the use of lenvatinib plus pembrolizumab in CCGCs.

Methods: This is a phase II, international, multi-center, single-arm trial of continuous daily oral lenvatinib $20 \mathrm{mg}$ plus intravenous pembrolizumab 200 mg 3-weekly. Patients with recurrent CCGC of either ovarian or endometrial origin after progression on at least one line of platinum-based chemotherapy, who have measurable disease and no prior immune checkpoint blockade are eligible. The primary endpoint of the trial is the objective response rate (ORR) by RECIST v1.1 criteria at 24 weeks. Secondary endpoints include ORR by iRECIST at 24 weeks, biochemical response rate, progressionfree survival rate at 24 weeks, clinical benefit rate at 24 weeks, duration of response, overall survival and adverse event profile. Translational multi-parametric immune analysis on archival tissue, pre-, on-treatment and post-progression tumor biopsies as well as blood samples will seek to explore prognostic and predictive biomarkers of response and resistance to this combination. Sample size calculations were based on the Simon 2-stage minimax design. Based on ORR up to 10\% (HO) in recurrent CCGC treated with chemotherapy and an alternate hypothesis of ORR up to 30\%, at a 5\% significance level (onesided alpha) and $80 \%$ power of rejecting $\mathrm{HO}, 15$ patients will be recruited to the first stage. If 2 or more patients demonstrate objective response, the study may proceed to full recruitment of 25 patients. LARA is currently open for accrual at three sites in Singapore and South Korea, and has 6 patients enrolled at the time of submission (NCT04699071, APGOT OV3). Trial Registration: ClinicalTrials.gov Identifier: NCT04699071

Poster (042)

Surgical Techniques \& perioperative Management

https://doi.org/10.3802/jgo.2021.32.S1.042

\section{Morbidity and mortality outcomes after cytoreductive surgery with hyperthermic intraperitoneal chemotherapy in a treatment of gynecologic malignancies}

\footnotetext{
Migang Kim, ${ }^{1}$ Ki Eun Seon, ${ }^{2}$ Yong Jae Lee, ${ }^{2}$ Jung-Yun Lee, ${ }^{2}$ Min Chul Choi ${ }^{1, *}$

'CHA Bundang Medical Center, Seongnam, Korea (oursk79@cha.ac.kr) ${ }^{2}$ Yonsei University College of Medicine, Seoul, Korea
}

Objective: It has been reported that hyperthermic intraperitoneal chemotherapy (HIPEC) with cytoreductive 
surgery (CRS) improved survival in selected patients with peritoneal carcinomatosis, although the procedures are associated with high morbidity and mortality rates. This study investigated the morbidity and mortality rate of CRS with HIPEC in patients with gynecologic cancers.

Methods: We reviewed retrospectively the medical records of patients who underwent CRS with HIPEC due to gynecologic cancer and pseudomyxoma peritonei from January 2013 to June 2021 at 2 institutions in Korea. The adverse events (AEs) that occurred within 60 days of HIPEC and operative factors related to the AEs were investigated.

Results: A total of 170 procedures were included in this study. There were 155 procedures with ovarian/tubal/peritoneal cancer (91.2\%) and $15(8.8 \%)$ with pseudomyxoma peritonei. The median age of patients was 55 years old (range, 16-79). The median score of the peritoneal carcinomatosis index was 9 (range, 0-29). The median operative time was 480 minutes (range, 195-1,080), and estimated blood loss was $600 \mathrm{~mL}$ (range, 50-8,600). AE of grade $\geq 3$ during hospitalization occurred in 63 procedures (37.1\%). The most common serious AE was anemia (34/170, 20\%), followed by neutropenia (4.7\%) and ileus $(4.1 \%)$. The mortality rate was $0.6 \%(1 / 170)$ due to acute myocardial infarction. A total of 29 patients $(17.1 \%)$ was re-hospitalization due to AEs within 60 days postoperative. Conclusion: The morbidity and mortality rates of CRS with HIPEC in gynecologic cancer patients are comparable with previous reports, and those are in acceptable rates even broad extent of surgery and the long operation time.

\section{Miscellaneous}

Poster (M01)

Quality of Life, Nursing \& Supportive Care

https://doi.org/10.3802/jgo.2021.32.S1.M01

\section{Factors influencing the use of complementary and alternative medicine in gynecologic oncology patients}

\section{Nuntorn Chukasemrat," Chuenkamon Charakorn, Arb-aroon Lertkhachonsuk}

Ramathibodi Hospital, Mahidol University, Bangkok, Thailand (ong_nuntorn@hotmail.com)

Objective: To determine the factors influencing the use of complementary and alternative medicine (CAM) in gynecologic cancer patients and the prevalence and pattern of CAM use. Methods: This was a cross-sectional study of 370 gynecologic cancer patients conducted at the out-patient clinic, Ramathibodi Hospital, Mahidol University, Bangkok, Thailand.
After informed consent, participants were asked to complete a standardized questionnaire including socio-demographic and clinical characteristic, detail of CAM use, attitude of CAM use, and quality of life using EORTC QLQ-C30.

Results: The prevalence of CAM use was $25.13 \%$. The most common type was herbal medicine $(55.90 \%)$. The participants who resided or had a birthplace in rural areas presented with a higher proportion of CAM use than those in urban areas $(\mathrm{p}=0.470$ and $\mathrm{p}=0.004$, respectively). Participants who received multiple modalities of cancer treatment reported significantly higher proportion of CAM use $(\mathrm{p}=0.024)$. Most CAM users agreed that the CAM could be used in combination with standard treatment, and some rather disagreed that CAM could interrupt the treatment effect of the conventional treatment. CAM users had significantly higher role functioning in qualityof-life scores.

Conclusion: Factors influencing CAM use in gynecologic cancer patients were rural area birthplace or residency, receiving multiple modalities of cancer treatment, having positive attitude towards CAM use. CAM users had better performance in role functioning in quality-of-life score. Therefore, gynecologic oncologists should pay attention to these factors in order to communicate with gynecologic cancer patients about CAM use.

Poster (M02)

Surgical Techniques \& Perioperative Management

https://doi.org/10.3802/jgo.2021.32.S1.M02

\section{Initial experience with the enhanced recovery after surgery (ERAS) protocols in gynecologic surgery at an urban academic tertiary medical center}

\author{
Won-Ji Kim, Joseph J. Noh, Yoo-Young Lee* \\ Samsung Medical Center, Seoul, Korea (yooyoung.lee@samsung.com)
}

Objective: The aim of this study was to describe the development and early experience of enhanced recovery after surgery (ERAS) protocols in gynecology at an urban academic medical center.

Methods: The target patient population included those who underwent major gynecologic surgeries for both benign and malignant diseases between October 2020 and January 2021. Two attending surgeons implemented the protocols for their patients (ERAS cohort) while 3 attending surgeons maintained conventional perioperative care for their patients (non-ERAS cohort). Baseline characteristics, surgical outcomes and patients' answers to a 12-question survey were compared. A case-matched comparative analysis was also performed between 